\title{
Discovery and progenitor constraints on the Type la supernova 2013gy ${ }^{\star}$
}

\author{
S. Holmbo ${ }^{1}$, M. D. Stritzinger ${ }^{1,2, \star \star}$, B. J. Shappee ${ }^{2}$, M. A. Tucker ${ }^{2}$, W. Zheng ${ }^{3}$, C. Ashall ${ }^{4}$, M. M. Phillips ${ }^{5}$,
} C. Contreras ${ }^{5}$, A. V. Filippenko ${ }^{3,6, \star \star \star}$, P. Hoeflich ${ }^{4}$, M. Huber ${ }^{2}$, A. L. Piro ${ }^{7}$, X. F. Wang ${ }^{8}$, J.-J. Zhang ${ }^{9,10}$, J. Anais ${ }^{5}$, E. Baron ${ }^{11,12, \star \star}$, C. R. Burns ${ }^{7}$, A. Campillay ${ }^{5}$, S. Castellón ${ }^{5}$, C. Corco ${ }^{13}$, E. Y. Hsiao ${ }^{4}$, K. Krisciunas ${ }^{14}$, N. Morrell ${ }^{5}$, M. T. B. Nielsen ${ }^{1}$, S. E. Persson ${ }^{7}$, F. Taddia ${ }^{15}$, L. Tomasella ${ }^{16}$, T.-M. Zhang ${ }^{17}$, and X.-L. Zhao ${ }^{8,18}$

1 Department of Physics and Astronomy, Aarhus University, Ny Munkegade 120, 8000 Aarhus C, Denmark e-mail: simonholmbo@phys.au.dk; max@phys.au.dk

2 Institute for Astronomy, University of Hawai'i, 2680 Woodlawn Drive, Honolulu, HI 96822, USA

3 Department of Astronomy, University of California, Berkeley, CA 94720-3411, USA

${ }^{4}$ Department of Physics, Florida State University, 77 Chieftain Way, Tallahassee, FL 32306, USA

5 Carnegie Observatories, Las Campanas Observatory, Casilla 601, La Serena, Chile

${ }^{6}$ Miller Institute for Basic Research in Science, University of California, Berkeley, CA 94720, USA

7 Observatories of the Carnegie Institution for Science, 813 Santa Barbara St., Pasadena, CA 91101, USA

8 Physics Department and Tsinghua Center for Astrophysics (THCA), Tsinghua University, Beijing 100084, PR China

9 Yunnan Observatories, Chinese Academy of Sciences, Kunming 650216, PR China

10 Key Laboratory for the Structure and Evolution of Celestial Objects, Chinese Academy of Sciences, Kunming 650216, PR China

11 Homer L. Dodge Department of Physics and Astronomy, University of Oklahoma, 440 W. Brooks, Rm 100, Norman, OK 73019-2061, USA

12 Visiting Astronomer, Hamburger Sternwarte, Gojenbergsweg 112, 21029 Hamburg, Germany

13 Cerro Tololo Inter-American Observatory, National Optical Astronomy Observatory, Casilla 603 La Serena, Chile

14 George P. and Cynthia Woods Mitchell Institute for Fundamental Physics and Astronomy, Department of Physics and Astronomy, Texas A\&M University, College Station, TX 77843, USA

15 The Oskar Klein Centre, Department of Astronomy, Stockholm University, AlbaNova, 10691 Stockholm, Sweden

16 INAF, Osservatorio Astronomico di Padova, 35122 Padova, Italy

17 National Astronomical Observatories of China, Beijing 100012, PR China

18 School of Science, Tianjin University of Technology, Tianjin 300384, PR China

Received 5 October 2018 / Accepted 30 January 2019

\section{ABSTRACT}

\begin{abstract}
We present an early-phase $g$-band light curve and visual-wavelength spectra of the normal Type Ia supernova (SN) 2013gy. The light curve is constructed by determining the appropriate S-corrections to transform KAIT natural-system $B$ - and $V$-band photometry and Carnegie Supernova Project natural-system $g$-band photometry to the Pan-STARRS1 $g$-band natural photometric system. A Markov chain Monte Carlo calculation provides a best-fit single power-law function to the first ten epochs of photometry described by an exponent of $2.16_{-0.06}^{+0.06}$ and a time of first light of MJD 56629. $4_{-0.1}^{+0.1}$, which is $1.93_{-0.13}^{+0.12}$ days (i.e., $<48 \mathrm{~h}$ ) before the discovery date (2013 December 4.84 UT) and $-19.10_{-0.13}^{+0.12}$ days before the time of $B$-band maximum (MJD $56648.5 \pm 0.1$ ). The estimate of the time of first light is consistent with the explosion time inferred from the evolution of the Si II $\lambda 6355$ Doppler velocity. Furthermore, discovery photometry and previous nondetection limits enable us to constrain the companion radius down to $R_{\mathrm{c}} \leq 4 R_{\odot}$. In addition to our early-time constraints, we used a deep +235 day nebular-phase spectrum from Magellan/IMACS to place a stripped H-mass limit of $<0.018 M_{\odot}$. Combined, these limits effectively rule out H-rich nondegenerate companions.
\end{abstract}

Key words. supernovae: general - supernovae: individual SN 2013gy

\section{Introduction}

Today, Type Ia supernova (SN Ia) cosmology is limited by a subtle matrix of systematic errors. Although significant effort is being placed to reduce known systematics in order to improve upon the accuracy of measuring their peak luminosities (see Phillips et al. 2019), obtaining distances accurate to the percent

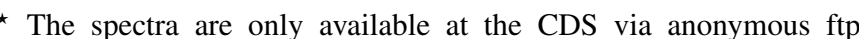
to cdsarc.u-strasbg. fr (130.79.128.5) or via http://cdsarc. u-strasbg.fr/viz-bin/qcat?]/A+A/627/A174.

$\star \star$ Visiting Astronomer.

$\star \star \star$ Miller Senior Fellow.
}

level will likely require a better understanding of their progenitors and explosion physics. Although SNe Ia are generally agreed to originate from the thermonuclear disruption of a carbon-oxygen white dwarf (Hoyle \& Fowler 1960), progenitor scenarios which give rise to $\mathrm{SNe} \mathrm{Ia}$ and explosion processes involved remain critical questions to answer. With the growth of wide field surveys, these questions can be addressed better than ever before. In particular, the discovery of SNe Ia shortly after explosion can provide new information through their early light curves, such as shock cooling of the white dwarf surface (Piro et al. 2010; Rabinak et al. 2012), collision 
with the companion star (Kasen 2010), evidence for shallow radioactive nickel (Piro \& Morozova 2016; Maeda et al. 2018; Polin et al. 2019), and interaction with circumstellar material (Piro \& Morozova 2016; Noebauer et al. 2016; Maeda et al. 2018; Tucker et al. 2019).

The current sample of SNe Ia with early-time observations exhibit at least two different morphologies (Stritzinger et al. 2018). In one case, the early light curves are well fit with a single power-law function. Objects exhibiting such a behavior include SN 2011fe (Zhang et al. 2016), KSN 2011b (Olling et al. 2015; Shappee et al. 2019), KSN 2012a (Olling et al. 2015; Shappee et al. 2019), ASASSN-14lp (Shappee et al. 2016), and SN 2015F (Im et al. 2015; Cartier et al. 2017). On the other hand, some SNe Ia exhibit a two-phase morphology. In such cases the first phase consists of a linear increase in flux typically extending over three to five days past the time of first light $\left(t_{\text {first }}\right)$, followed by an abrupt increase in flux (power law) as the light curve rises to maximum brightness. Objects exhibiting a two-phase morphology include SN 2012cg, (Marion et al. 2016; Shappee et al. 2018), SN 2012fr (Contreras et al. 2018), SN 2013dy (Zheng et al. 2013; Pan et al. 2015), SN 2014J (Zheng et al. 2014; Siverd et al. 2015; Goobar et al. 2015), iPTF 16abc (Miller et al. 2018), and SN 2017cbv (Hosseinzadeh et al. 2017). A two-component power-law function (or broken power-law function; Zheng et al. 2017) provides superior fits to the early light curves of these objects and more accurate estimates of both $t_{\text {first }}$ and the rise time $\left(t_{\text {rise }}\right)$ to maximum $\left(t_{\text {max }}\right)$.

Aside from four SNe Ia located in Kepler fields (Olling et al. 2015; Brown et al. 2018; Cornect et al. 2018), the current sample of objects studied at early times consists of low-cadence coverage, and it may be possible that a two-component emission structure has been misinterpreted as a single power law. For example, Foley et al. (2012) fit the early light curve of SN 2009ig with a single power law; however, when photometry computed from an open-filter discovery image is included, a two-component power law may indeed provide a superior fit (see Contreras et al. 2018). Taken together with the recent observations of MUSSES1604D (also known as SN 2016jhr; Jiang et al. 2017) a SN 2006bt-like event (Foley et al. 2010; Stritzinger et al. 2011) that exhibited clear departures from a single and double power-law rise, the early-phase observational parameter space of SNe Ia is far from fully explored.

Currently there are $<20 \mathrm{SNe}$ Ia in the literature discovered within approximately three days of $t_{\text {first }}$, and the addition of more objects to the sample is crucial to fully map out the early-time parameter space. Here we present an early $g$-band light curve of the normal SN 2013gy (also known as PS1-13ejo) based on photometry calibrated to one well-understood photometric system. Specifically, Pan-STARRS1 (PS1; Tonry et al. 2012; Chambers et al. 2016) first $g$-band detection images are combined with follow-up imaging obtained by the Carnegie Supernova Project-II (CSP-II; Phillips et al. 2019) and the Lick Observatory Supernova Search (LOSS; Filippenko et al. 2001). In addition to the light curve, a comprehensive set of visualwavelength spectra is also presented.

Kim et al. (2013) announce the discovery of SN 2013gy in open-filter images obtained with the $0.76 \mathrm{~m}$ Katzman Automatic Imaging Telescope (KAIT) at Lick Observatory on 2013 December 6.33 (UT dates are used throughout this paper). An openfilter image taken the previous day provides a nondetection limit of $19.3 \mathrm{mag}$. SN 2013gy was also recovered in PS1 images obtained on 2013 December 5.34 with an apparent $g$-band (hereafter $g_{\mathrm{PS} 1}$ ) magnitude of $19.48 \pm 0.09$. The previous PS1 nondetection image was obtained 5.95 days earlier on 2013
November 29.39 with a limiting $g_{\mathrm{PS} 1}$ magnitude of 20.54. An optical spectrum obtained by Tomasella et al. (2013) on 2013 December 7.5 indicated the object was a young SN Ia.

With $\mathrm{J} 2000$ coordinates of $\alpha=03^{\mathrm{h}} 42^{\mathrm{m}} 16.88$ and $\delta=$ $-04^{\circ} 43^{\prime} 18^{\prime \prime} 52$, SN 2013 gy was located $32^{\prime \prime}$ north and $11^{\prime \prime}$ east from the center of the SB(s)b host galaxy NGC 1418. A color image of NGC 1418 with the position of SN 2013gy indicated is provided in Fig. A.1. The redshift of NGC 1418 is $z=$ 0.014023 (Catinella et al. 2005) and its Tully Fisher (TF) distance ranges from $43.8 \mathrm{Mpc}$ (Springob et al. 2007) to $88.8 \mathrm{Mpc}$ (Theureau et al. 2007). Given the high dispersion of the TF distances, in the following we adopt the redshift distance, which after correcting for a Virgo, Great Attractor, and Shapley infall model and adopting $H_{0}=73 \mathrm{~km} \mathrm{~s}^{-1} \mathrm{Mpc}^{-1}$ corresponds to a distance of $55.9 \pm 3.9 \mathrm{Mpc}$ (i.e., $\mu=33.75 \pm 0.15 \mathrm{mag}$ ). This is fully consistent with the distance derived from the comprehensive set of optical and near-infrared (NIR) light curves obtained by the CSP-II, which provides $\mu=33.68 \pm 0.09 \mathrm{mag}$ (see Sect. 2.3).

\section{Early-time observations of SN 2013gy}

\subsection{Photometry and visual-wavelength spectroscopy}

Our early-phase $g_{\text {PS1 }}$-band light curve of SN 2013gy is constructed using data from three different facilities. This includes two epochs of $g_{\mathrm{PS} 1}$ separated by $\sim 20 \mathrm{~min}, 52$ epochs of $g$-band photometry obtained by the CSP-II (hereafter $g_{\mathrm{CSP}}$ ) extending from $-15.3 \mathrm{~d}^{1}$ to $+59.4 \mathrm{~d}$, and 37 epochs of $B$ - and $V$-band photometry from KAIT extending from $-16.2 \mathrm{~d}$ to $+53.6 \mathrm{~d}$. To facilitate our analysis presented below, in the following the KAIT $B$ - and $V$-band photometry is summed together (in flux space) and then multiplied by one half, creating what we refer to as $B V .5$-band photometry (see below). Reduced $g_{\mathrm{PS} 1}$-band photometry was downloaded from the PS1 webpage ${ }^{2}$, while CSP-II and KAIT images were reduced in the standard manner following the techniques described by Krisciunas et al. (2017) and Zheng et al. (2013), respectively. Natural-system photometry of the supernova was computed for each set of science images relative to local sequences of stars. These local sequences of stars were calibrated to the natural system of each setup using standard-star photometry converted to the natural systems through the use of color terms obtained from multiple observations of the standard fields (see Contreras et al. 2018, for details). The resulting $g_{\mathrm{PS} 1^{-}}$, $g_{\mathrm{CSP}^{-}}$, and KAIT $B V .5$-band photometry is listed in Table A.1, along with the S-corrections (see below) that transform their photometry to the $g_{\mathrm{PS} 1}$ natural system.

Table A.2 contains the journal of spectroscopic observations. These include 18 epochs of unpublished early optical spectra, five published spectra (Graham et al. 2017), and a latephase spectrum taken with the Magellan Baade telescope. The spectra were reduced following standard procedures (see, e.g., Hamuy et al. 2006), were color-matched to multiband photometry, and are plotted in the rest frame in Fig. A.2.

\subsection{S-correction and a definitive $g_{P S 1}$ light curve}

Here we describe how the $g_{\mathrm{CSP}^{-}}$and $B V .5$-band photometry was transformed to the $g_{\mathrm{PS} 1}$ natural system. We transform to the $g_{\mathrm{PS} 1}$ natural system to avoid S-correcting the earliest

\footnotetext{
1 In keeping with tradition, temporal phases are given with respect to the time of $B$-band maximum brightness $\left(t_{B, \max }\right)$ unless explicitly stated. 2 https://star.pst.qub.ac.uk/ps1threepi/psdb/ candidate/1034216880044318600/ (Smartt et al. 2014).
} 

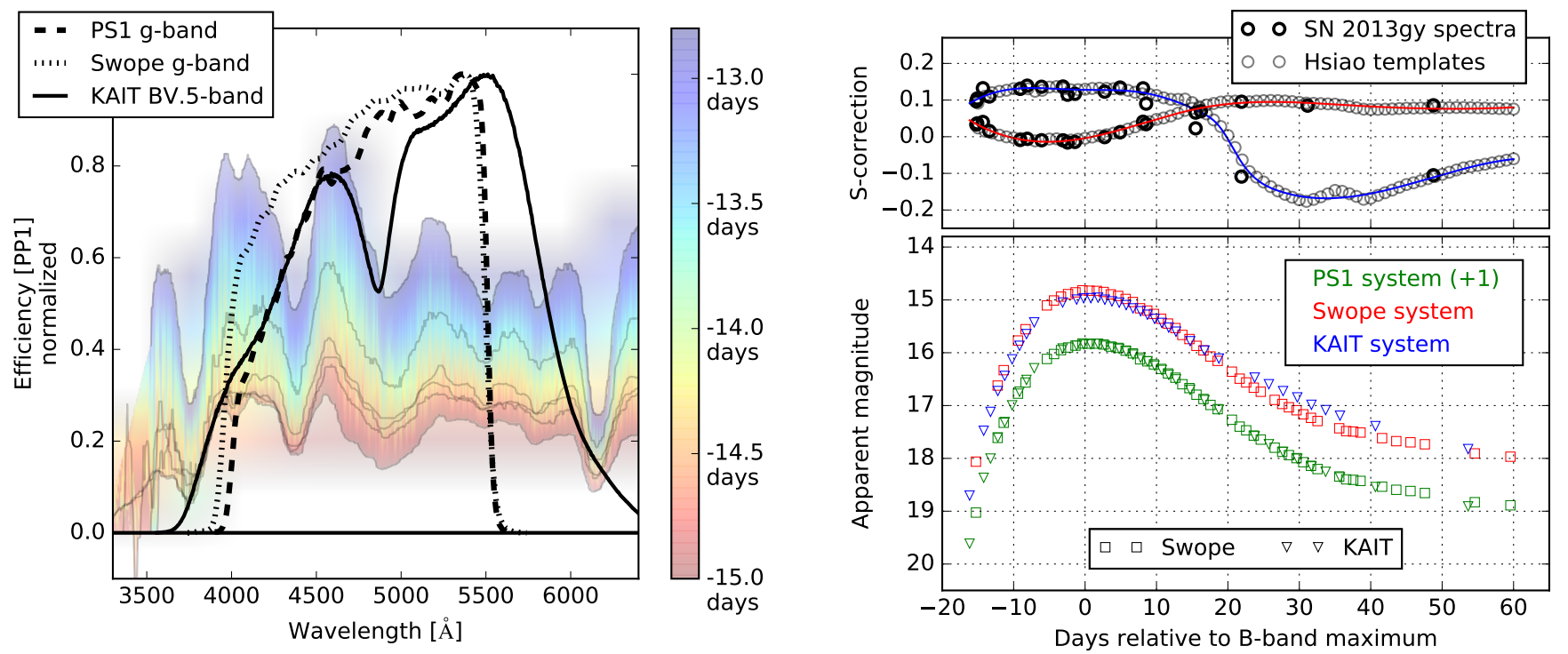

Fig. 1. System response functions and S-correction results. Left: comparison of the normalized $g_{\mathrm{PS} 1}$ (dashed line), the $g_{\mathrm{CSP}}$ (dotted line), and $B V .5$ (solid line) system response functions. Also plotted are seven visual-wavelength spectra of SN 2013gy ranging between $-16.1 \mathrm{~d}$ and $-13.4 \mathrm{~d}$ as indicated by the color bar. Right, top panel: S-corrections as a function of phase using observed (dark circles) spectra and Hsiao et al. (2007) template spectra (light circles). The smoothing splines of those S-corrections (red and blue lines for $g_{\text {CSP }}$ and $B V .5$, respectively) enable us to accurately transform $g_{\mathrm{CSP}}$-band and $B V .5$-band natural-system photometry to the $g_{\mathrm{PS} 1}$ natural system at any phase. Right, bottom panel: $g_{\mathrm{CSP}}$ (red squares) and $B V .5$-band (blue triangles) natural-system photometry, and the S-corrected versions (green squares and green triangles) transformed to the $g_{\mathrm{PS} 1}$ natural system (offset by $+1 \mathrm{mag}$ ).

observations where the S-corrections are more uncertain. To compute accurate $\mathrm{S}$-corrections requires system response functions and a spectrum, and/or a spectral template (Stritzinger et al. 2002). The left panel of Fig. 1 contains the $g_{\mathrm{PS}^{-}}, g_{\mathrm{CSP}^{-}}$, and KAIT $B V .5$-band response functions, along with early-phase optical spectra. The $B V .5$ response function is obtained by summing together the KAIT $B$ - and $V$ - response functions and multiplying the product by one half. The motivation for doing this is that the S-corrections computed for the KAIT $B$ and $V$ bands are found to be very similar (on the order of $\pm 0.6 \mathrm{mag}$ ), but with opposite signs. So, by combining their photometry into a $B V .5$ system, the required S-corrections to transform the KAIT photometry to the $g_{\text {PS }}$ system are minimized and found to lie between -0.1 to 0.2 mag out to $+60 \mathrm{~d}$.

To compute an S-correction at a specific epoch, the observed and template spectra are first color-matched to CSP-II broad-band photometry. The S-correction that transforms $g_{\mathrm{CSP}}$-band naturalsystem photometry to the $g_{\mathrm{PS} 1}$-band natural system is computed by taking the difference between synthetic photometry computed using the two different passbands and the spectrum - that is, $m\left(g_{\mathrm{PS} 1}\right)=m\left(g_{\mathrm{CSP}}\right)-\Delta \mathrm{S}$. Given that the spectral energy distribution of $\mathrm{SNe}$ Ia is time dependent, $\mathrm{S}$-corrections are computed for each epoch and then a smoothing spline is derived to give $\mathrm{S}$-corrections at all times. Imaging was obtained using both the observed spectra and the Hsiao et al. spectral template. The Scorrections transforming our $g_{\mathrm{CSP}}-$ and $B V .5$-band photometry to the $g_{\mathrm{PS} 1}$ natural system are plotted in Fig. 1 (right, top panel) and listed in Table A.1. The observed $g_{\mathrm{CSP}}$ and $B V .5$ photometry is also plotted (right, bottom panel) with and without the S-corrections.

\subsection{Light-curve parameters and reddening}

Basic light-curve parameters were computed using the lightcurve fitter SNooPy (Burns et al. 2011), and key results are listed in Table A.3. SNooPy fits provide an estimate of the time and magnitude of peak brightness, the light-curve decline- rate parameter $\Delta m_{15}(B)$, and the color-stretch parameter $s_{B V}$ (Burns et al. 2014), as well as host-galaxy reddening. Using the SNooPy "max model" $t_{B, \max }$ is found to have occurred on MJD $=56648.5 \pm 0.1$ with $m_{B}=14.699 \pm 0.013 \mathrm{mag}$, and $\Delta m_{15}(B)=1.234 \pm 0.060 \mathrm{mag}$. Fitting the entire suite of CSP-II optical/NIR light curves ${ }^{3}$ with SNooPy's "EBV method2", we obtain an $s_{B V}=0.892 \pm 0.05 \mathrm{mag}$, a host-galaxy color excess of $E(B-V)_{\text {host }}=0.106 \pm 0.004_{\text {stat }} \pm 0.060_{\text {sys }}$ mag, and a distance modulus $\mu=33.68 \pm 0.09$ mag. Combining $E(B-V)_{\text {host }}$ with the Milky Way component of $E(B-V)_{M W}=0.049 \mathrm{mag}$ (Schlafly \& Finkbeiner 2011; Schlegel et al. 1998), we obtain $E(B-V)_{\text {tot }}=0.155 \pm 0.060 \mathrm{mag}$, which is adopted throughout. Adjusting $m_{B}$ for reddening and K-corrections, and assuming our adopted distance to the host, we find that SN 2013gy reached a peak absolute $B$-band magnitude $M_{B}=-19.32 \pm 0.16$ mag.

\section{Results}

\subsection{Early $g_{P S 1}$ light curve and constraints on $t_{\text {first }}$}

Plotted in the left panel of Fig. 2 is the early-time $g_{\mathrm{PS} 1}$-band light curve of SN 2013gy. Overplotted on the light curve is the best-fit single power-law function (i.e., $\left.f(t)=A\left(t-t_{\text {first }}\right)^{\alpha}\right)$, a single power-law function with an index of two, and a linear function. The right, top panel shows the residuals between the best-fit model and the photometry. Finally, the bottom right panel of Fig. 2 contains the estimated error in the fit parameters and their correlation. Fitting was performed using the Markov chain Monte Carlo (MCMC) Python package PyMC ${ }^{4}$ (Salvatier et al. 2016) and the No-U-Turn Sampler (NUTS; Hoffman \& Gelman 2011). When fitting we considered only data obtained prior to $-10 \mathrm{~d}$ (Conley et al. 2006; Ganeshalingam et al. 2011), and $\alpha$ was a free parameter with priors defined using the results of

\footnotetext{
3 A detailed analysis of a comprehensive ultraviolet, optical, and NIR dataset of SN 2013gy will be presented in a forthcoming publication.

4 https://pymc-devs.github.io/pymc3
} 

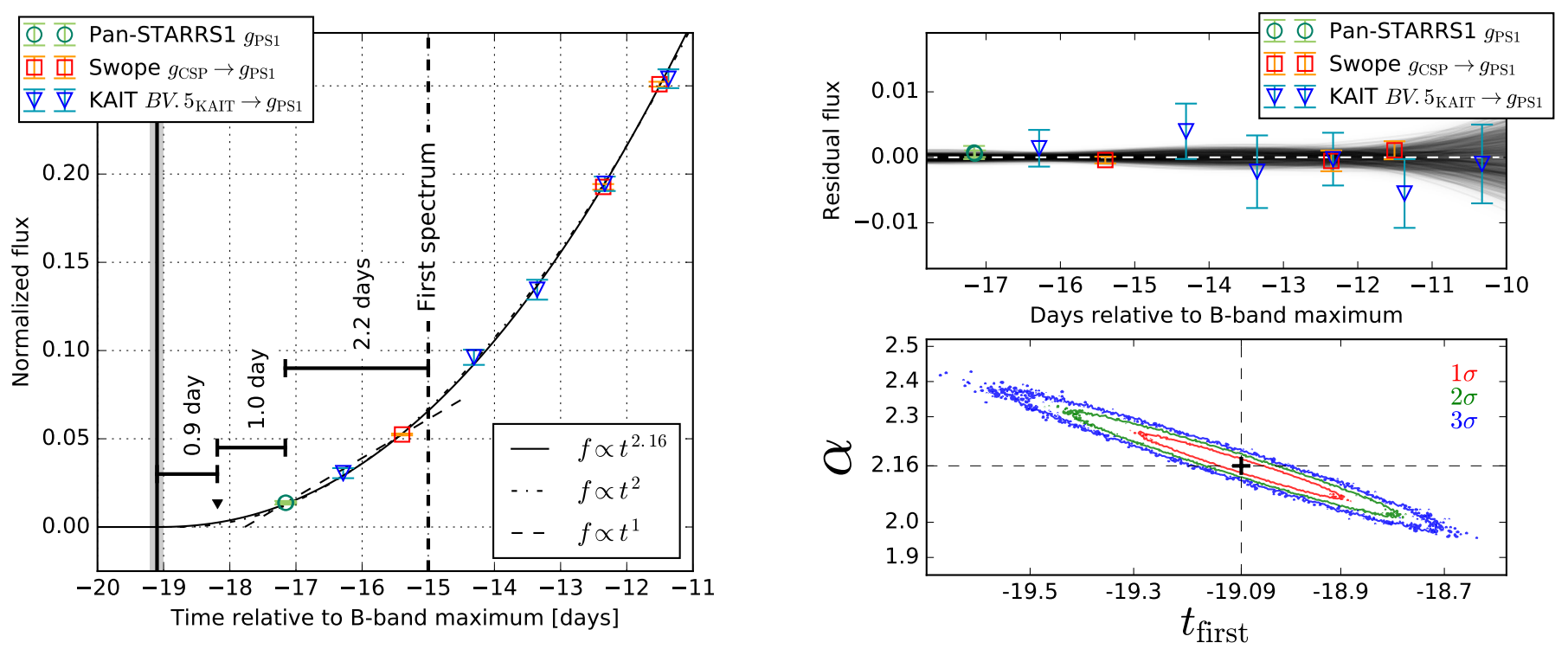

Fig. 2. Left: early-time $g_{\mathrm{PS} 1}$-band light curve of SN 2013gy based on photometry measured from PS1, CSP-II, and KAIT images, plotted along with the best-fit, single power-law function characterized by an index $\alpha=2.16$ (solid curve), the "fireball" model ( $\alpha=2$, dash-dot curve), and a linear rise for the first $\sim 3 \mathrm{~d}$ (dashed curve). The KAIT open-filter nondetection obtained $1 \mathrm{~d}$ prior to discovery is plotted as a black filled triangle. The solid vertical black line indicates $t_{\text {first }}$ with its width corresponding to the $1 \sigma$ uncertainty (gray region), and the vertical dashed line corresponds to the epoch of the first spectrum. Right, top: residuals between the best-fit model and the data (horizontal dashed white line); black lines correspond to models sampled from below (right, bottom panel), which contains $1 \sigma, 2 \sigma$, and $3 \sigma$ error ellipses indicating the uncertainties in the model fit parameters $\alpha$ and $t_{\text {first }}$.

Firth et al. (2015). The best-fit power law corresponds to $\alpha=$ $2.16_{-0.06}^{+0.06}$ and $t_{\text {first }}=56629.4_{-0.1}^{+0.1}$ MJD. We note that from examination of the right, bottom panel of Fig. 2, a value of $\alpha=2.0$ is found to be within $2 \sigma$ of the best-fit value. Assuming $\alpha=2.0$ would imply a $t_{\text {first }}$ value that is $7 \mathrm{~h}$ later than our inferred bestfit value. We also note that because the first 3 epochs span more than three days and the uncertainty of the first KAIT measurement is relatively large, we cannot rule out a linear rise prior to $\sim 15 \mathrm{~d}$ before $t_{B, \max }$. The best fit to a linear rise implies a value of $t_{\text {first }}$ that is $1.3 \mathrm{~d}$ later and only $14 \mathrm{~h}$ before the PS1 images. Comparison of the best-fit $t_{\text {rise }}$ to the first PS1 detection indicates that SN 2013gy was discovered $1.93_{-0.13}^{+0.12} \mathrm{~d}$ past $t_{\text {first }}$, and when compared to $t_{B, \max }$, one obtains $t_{\text {rise }}=-19.10_{-0.13}^{+0.12}$ days.

\subsection{Spectroscopy and an estimate of $t_{\text {explosion }}$}

Spectra of SN 2013gy taken around maximum light resemble those of normal SNe Ia. Pseudo-equivalent-width (pEW) measurements of the Si II $\lambda 5972$ anda superior fit and implies $\lambda 6355$ features indicate that it is "core normal" $(\mathrm{CN})$ according to Branch et al. (2006). Moreover, the position of the absorption minimum of the Si II $\lambda 6355$ feature in the $-0.8 \mathrm{~d}$ spectrum indicates a Doppler velocity at maximum absorption of $-v_{\mathrm{abs}}=10180 \pm 90 \mathrm{~km} \mathrm{~s}^{-1}$, consistent with a normal object in the Wang et al. (2009) system.

Piro \& Nakar (2013) suggest that an estimate of the explosion time $\left(t_{\text {explosion }}\right)$ can be obtained through the evolution of Si II 16355. This is achieved by fitting the early-time velocity evolution with an appropriate power law. Following Piro \& Nakar, we fit a $v \propto t^{\beta}$ power law with $\beta=-0.20,-0.22$, and -0.24 . The resulting goodness-of-fit parameter $\chi^{2}$ per degree of freedom $\left(\chi^{2} /\right.$ d.o.f. $)$ is plotted in the top-left panel of Fig. 3 for each power-law index. A constant systematic error is added to get a best fit with $\chi^{2} /$ d.o.f. $=1$. We find that $\beta=-0.20$ provides a superior fit and implies $t_{\text {explosion }}=56629.45_{-0.31}^{+0.28}$ MJD.
Comparison of $t_{\text {first }}$ and $t_{\text {explosion }}$ reveals that SN 2013gy likely experienced minimal to no dark phase.

\subsection{Constraints on interaction with a nondegenerate companion}

Companion radius $\left(R_{\mathrm{c}}\right)$ limits are computed following the methodology of Shappee et al. (2018), which is based on comparing the observations to the analytical model predictions of Kasen (2010). Taking a conservative explosion time to be $\sim 2 \mathrm{~d}$ before discovery, mass of the companion to be $1 M_{\odot}$ and assuming a favorable viewing angle of $15^{\circ}$, we compute $R_{\mathrm{c}}$ limits using both the discovery $g_{\mathrm{PS} 1}$ photometry and the previous KAIT nondetection. Our limits on $R_{\mathrm{c}}$ are plotted vs. days relative to the epoch of discovery in the topright panel of Fig. 3. Combined limits indicate $R_{\mathrm{c}} \lesssim 4 R_{\odot}$ in the case of a favorable viewing angle. Adopting the TF distances as upper and lower bounds on the distance, results in $R_{\mathrm{c}} \sim 3.4_{-2.1}^{+8.5} R_{\odot}$ for the estimated value of $t_{\text {first }}$. The same analysis has been done a number of times for other $\mathrm{SNe}$, yielding possible companion radii limits ranging between a fraction of $R_{\odot}$ and tens of $R_{\odot}$ (Bloom et al. 2012; Foley et al. 2012; Silverman et al. 2012; Goobar et al. 2015; Olling et al. 2015; Im et al. 2015; Marion et al. 2016; Shappee et al. 2016, 2018; Hosseinzadeh et al. 2017; Miller et al. 2018).

\subsection{Limits on the presence of companion material}

Hydrodynamic simulations of ejecta-companion interactions indicate that $>0.15 M_{\odot}$ of material could be removed from hydrogen-rich (H-rich) Roche-lobe overflow (RLOF) companions via stripping and/or momentum-transfer or ablation and/or heating and is expected to have a velocity dispersion of $v \approx 1000 \mathrm{~km} \mathrm{~s}^{-1}$ (e.g., Marietta et al. 2000; Pan et al. 2012; Boehner et al. 2018). This unbound material should be visible 

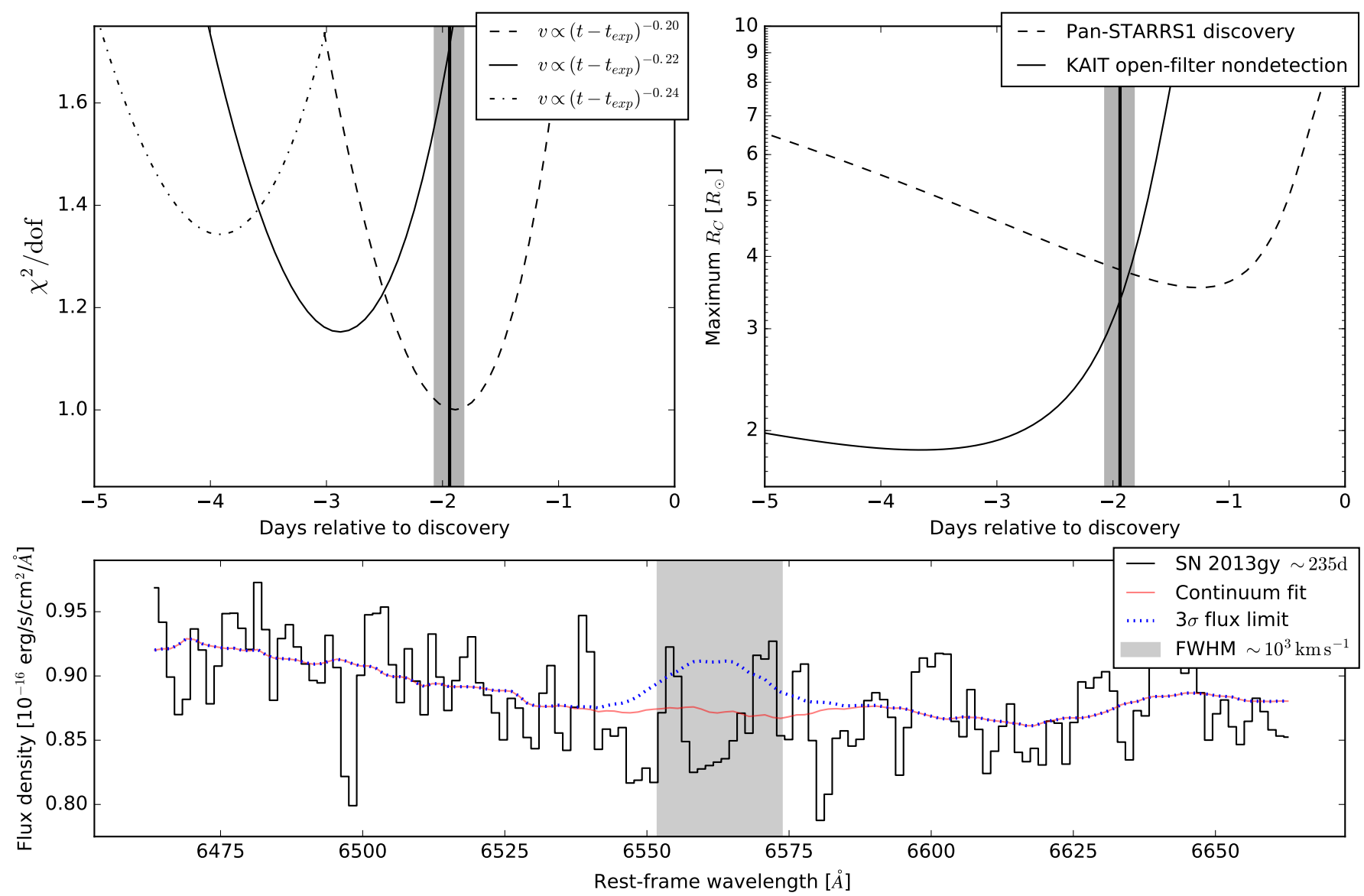

Fig. 3. Left: estimate of $t_{\text {explosion }}$ based upon functional fits to the velocity evolution of Si II $\lambda 6355$. Right: maximum allowed radius of a companion star as a function of explosion time as inferred from the discovery $g_{\mathrm{PS} 1}$ photometry and the previous KAIT nondetection limit plotted vs. days since discovery. The vertical black line in the left and right panels corresponds to the best-fit $t_{\text {first }}$, accompanied by a shaded gray region corresponding to its $1 \sigma$ uncertainty. Bottom: rest-frame-corrected nebular spectrum of SN 2013gy (black line), the continuum fit (red), and our $3 \sigma \mathrm{H} \alpha$ flux limit (blue line). The shaded area extends over the expected location of $\mathrm{H} \alpha \pm 1000 \mathrm{~km} \mathrm{~s}^{-1}$.

during the nebular phase - when the ejecta become optically thin - and lead to a prominent emission signature.

To place a limit on the $\mathrm{H} \alpha$ emission we have followed conventional techniques in the literature (e.g., Leonard 2007; Shappee et al. 2013). Briefly, the late-phase $(+235 \mathrm{~d})$ spectrum of SN 2013gy was flux calibrated to match photometry computed from PS1 stars (Flewelling et al. 2016) located in an $R$-band acquisition image. Next, the continuum was fitted in the vicinity of $\mathrm{H} \alpha$ and subtracted from the spectrum, yielding no statistical evidence for $\mathrm{H} \alpha$ emission as demonstrated in the bottom panel of Fig. 3. Following Leonard (2007), we obtain a $3 \sigma$ statistical flux limit of $4.5 \times 10^{-16} \mathrm{ergs} \mathrm{s}^{-1} \mathrm{~cm}^{-2} \AA^{-1}$. For our adopted distance and using the models of Botyánszki et al. (2018), this corresponds to an $\mathrm{H}$-mass limit of $<0.018 M_{\odot}$. This limit is an order of magnitude lower than all estimates of unbound mass values for normal $\mathrm{SNe}$ Ia, effectively ruling out $\mathrm{H}$-rich RLOF companions. This finding is consistent with similar constraints of a number of other SNe Ia discovered soon after explosion (Mattila et al. 2005; Leonard 2007; Lundqvist et al. 2013, 2015; Shappee et al. 2013, 2018; Maguire et al. 2016; Graham et al. 2017; Sand et al. 2018; Dimitriadis et al. 2019). This leaves only a small parameter space of subdwarfs and He stars whose signatures could remain undetected in the spectrum. We note that inspection of an unpublished early-phase NIR spectral time-series of SN2013gy obtained by the CSP-II yields no indication of He lines, and unfortunately, no late-phase NIR spectrum was obtained.

\section{Discussion}

We have presented discovery and follow-up photometry of the normal SN Ia 2013gy. Photometry obtained from different facilities was carefully calibrated to the $g_{\mathrm{PS} 1}$ system. The early rise of the light curve is well fit with a single power-law function, indicating that SN 2013gy was discovered within $48 \mathrm{~h}$ of $t_{\text {first }}$. The corresponding $t_{\text {first }}$ value is consistent with a $t_{\text {explosion }}$ estimate based on the Doppler velocity evolution of the Si II $\lambda 6355$ feature, suggesting minimal to no dark phase for SN 2013gy. A short dark phase has been inferred from the study of several other supernovae including SN 2009ig, SN 2011fe, SN 2012cg (Piro \& Nakar 2014), and iPTF 16abc (Miller et al. 2018). In contrast, the transitional iPTF 13ebh may have experienced an approximately four-day dark phase (see Hsiao et al. 2015).

Examination of the pEW values of the Si II $\lambda 5972$ and $\lambda 6355$ doublets shows that SN 2013gy is core normal (Branch et al. 2006, 2009), while its Si II $\lambda 6355$ Doppler velocity makes it a normal object according to the classification scheme of Wang et al. (2009). Moreover, as discussed by Stritzinger et al. (2018), the early intrinsic $(B-V)_{0}$ colors reveal it to be a red and rapidly evolving object, similar to SN 2011fe. In summary, SN 2013gy displays all of the characteristics of a normal SN Ia.

Making use of the early discovery of SN 2013gy, limits were placed on the radius of any companion star of $R \leq$ 
$4 M_{\odot}$. Combined with our stripped H-mass limit of $\lesssim 0.018 M_{\odot}$ obtained from a nebular-phase Magellan spectrum, our analysis effectively rules out H-rich RLOF companions. This result highlights that it is possible to constrain existing SN Ia progenitor models with the use of both early and late-phase observations.

Acknowledgements. A special thanks to the referee for detailed comments to the submitted manuscript. We thank P. Massey and M. Kasliwal for obtaining a spectrum each with the Magellan telescopes. S. Holmbo and M. D. Stritzinger are supported by a research grant (13261) from the VILLUM FONDEN A. V. Filippenko's supernova group is grateful for financial assistance from USA National Science Foundation (NSF) grant AST-1211916, the TABASGO Foundation, the Christopher R. Redlich Fund, and the Miller Institute for Basic Research in Science (U. C. Berkeley). Research at Lick Observatory is partially supported by a generous gift from Google. P. Höflich acknowledges NSF grants AST-1715133 and AST-1613472. L. Tomasella is partially supported by the PRIN-INAF 2016 with the project "Towards the SKA and CTA era: discovery, localisation, and physics of transient sources" (P.I. M. Giroletti). The CSP-II has been funded by the USA NSF under grants AST-0306969, AST0607438, AST-1008343, AST-1613426, AST-1613455, and AST-1613472, and in part by a Sapere Aude Level 2 grant funded by the Danish Agency for Science and Technology and Innovation (PI Stritzinger). J.-J. Zhang is supported by the National Natural Science Foundation of China (NSFC, grants 11403096 and 11773067), the Key Research Program of the CAS (grant KJZDEW-M06), the Youth Innovation Promotion Association of the CAS (grant 2018081), and the CAS Light of West China Program. X. Wang is supported by the National Natural Science Foundation of China (NSFC grants 11325313 and 11633002), and the National Program on Key Research and Developmen Project (grant 2016YFA0400803). Based in part on observations made with the Nordic Optical Telescope (P49-017; PI Stritzinger), operated by the Nordic Optical Telescope Scientific Association at the Observatorio del Roque de los Muchachos, La Palma, Spain, of the Instituto de Astrofisica de Canarias. This paper includes data gathered with the $6.5 \mathrm{~m}$ Magellan telescopes located at Las Campanas Observatory, Chile. We acknowledge the support of the staff of Lijiang $2.4 \mathrm{~m}$ and the Xinglong $2.16 \mathrm{~m}$ telescopes. Partially based on observations made with the Copernico $1.8 \mathrm{~m}$ telescope (Asiago, Italy) operated by INAF Osservatorio Astronomico di Padova. This paper made use of discovery image obtained by the Pan-STARRS1 Survey (PS1), which was made possible through contributions of the Institute for Astronomy, the University of Hawaii, the PanSTARRS Project Office, the Max-Planck Society and its participating institutes, the Max Planck Institute for Astronomy, Heidelberg and the Max Planck Institute for Extraterrestrial Physics, Garching, The Johns Hopkins University, Durham University, the University of Edinburgh, Queen's University Belfast, the Harvard-Smithsonian Center for Astrophysics, the Las Cumbres Observatory Global Telescope Network Incorporated, the National Central University of Taiwan, the Space Telescope Science Institute, the National Aeronautics and Space Administration (NASA) under grant NNX08AR22G issued through the Planetary Science Division of the NASA Science Mission Directorate, the NSF under grant AST-1238877, the University of Maryland, and Eotvos Lorand University (ELTE).

\section{References}

Bloom, J. S., Kasen, D., Shen, K. J., et al. 2012, ApJ, 744, L17 Boehner, P., Plewa, T., \& Langer, N. 2018, MNRAS, 465, 2060 Botyánszki, J., Kasen, D., \& Plewa, T. 2018, ApJ, 852L, 6 Branch, D., Dang, L. C., \& Hall, N. 2006, PASP, 118, 560 Branch, D., Dang, L. C., \& Baron, E. 2009, PASP, 121, 238 Brown, J. S., Stanek, K. Z., Vallely, P., et al. 2018, ATel, 11253, 00 Burns, C. R., Stritzinger, M. D., Phillips, M. M., et al. 2011, AJ, 141, 19 Burns, C. R., Stritzinger, M. D., Phillips, M. M., et al. 2014, ApJ, 789, 32 Cartier, R., Sullivan, M., Firth, R. E., et al. 2017, MNRAS, 464, 4476 Catinella, B., Haynes, M. P., \& Giovanelli, R. 2005, AJ, 130, 1037

Chambers, K. C., Magnier, E. A., \& Metcalfe, N. 2016, ArXiv e-prints [arXiv:1612.05560]

Conley, A., Howell, D. A., Howes, A., et al. 2006, AJ, 132, 1707

Contreras, C., Phillips, M. M., Burns, C. R., et al. 2018, ApJ, 859, 24

Cornect, R., Brimacombe, J., Stone, G., et al. 2018, ATel, 11259

Dimitriadis, G., Rojas-Bravo, C., Kilpatrick, C. D., et al. 2019, ApJ, 870, L14
Filippenko, A. V., Li, W. D., Treffers, R. R., \& Modjaz, M. 2001, in Small Telescope Astronomy on Global Scales, eds. W. P. Chen, C. Lemme, \& B. Paczyński (San Francisco: ASP), 246, 121

Firth, R. E., Sullivan, M., Gal-Yam, A., et al. 2015, MNRAS, 446, 3895

Flewelling, H. A., Magnier, E. A., \& Chambers, K. C. 2016, ArXiv e-prints [arXiv:1612.05243]

Foley, R. J., Narayan, G., Challis, P. J., et al. 2010, ApJ, 708, 1748

Foley, R. J., Challis, P. J., Filippenko, A. V., et al. 2012, ApJ, 744, 38

Ganeshalingam, M., Li, W., \& Filippenko, A. V. 2011, MNRAS, 416, 2607

Goobar, A., Kromer, M., Siverd, R., et al. 2015, ApJ, 799, 106

Graham, M. L., Kumar, S., Hosseinzadeh, G., et al. 2017, MNRAS, 472, 3437

Hamuy, M., Folatelli, G., Morrell, N. I., et al. 2006, PASP, 118, 2

Hoffman, M. D., \& Gelman, A. 2011, ArXiv e-prints [arXiv:1111.4246]

Hosseinzadeh, G., Sand, D. J., Valenti, S., et al. 2017, ApJ, 845, L11

Hoyle, F., \& Fowler, W. A. 1960, ApJ, 132, 565

Hsiao, E. Y., Conley, A., Howell, D. A., et al. 2007, ApJ, 663, 1187

Hsiao, E. Y., Burns, C. R., Contreras, C., et al. 2015, A\&A, 578, A9

Im, M., Choi, C., Yoon, S.-C., et al. 2015, ApJS, 221, 22

Jiang, J., Doi, M., Maeda, K., et al. 2017, Nature, 550, 80

Kasen, D. 2010, ApJ, 708, 1025

Kim, H., Zheng, W. K., Li, W., et al. 2013, CBET, 3743, 1

Krisciunas, K., Contreras, C., Burns, C. R., et al. 2017, AJ, 154, 211

Leonard, D. C. 2007, ApJ, 670, 1275

Lundqvist, P., Mattila, S., Sollerman, J., et al. 2013, MNRAS, 435, 329

Lundqvist, P., Nyholm, A., Taddia, F., et al. 2015, A\&A, 577, A39

Maeda, K., Jiang, J.-A., Shigeyama, T., \& Doi, M. 2018, ApJ, 861, 78

Maguire, K., Taubenberger, S., Sullivan, M., \& Mazzali, P. A. 2016, MNRAS, 457, 3254

Marietta, E., Burrows, A., \& Fryxell, B. 2000, ApJS, 128, 615

Marion, G. H., Brown, P. J., Vinkó, J., et al. 2016, ApJ, 820, 92

Mattila, S., Lundqvist, P., Sollerman, J., et al. 2005, A\&A, 443, 649

Miller, A. A., Cao, Y., Piro, A. L., et al. 2018, ApJ, 852, 100

Noebauer, U. M., Taubenberger, S., Blinnikov, S., Sorokina, E., \& Hillebrandt, W. 2016, MNRAS, 463, 2972

Olling, R. P., Mushotzky, R., Shaya, E. J., et al. 2015, Nature, 521, 332

Pan, K.-C., Ricker, P. M., \& Taam, R. E. 2012, ApJ, 760, 21

Pan, Y.-C., Foley, R. J., Kromer, M., et al. 2015, MNRAS, 452, 4307

Phillips, M. M., Contreras, C., Hsiao, E. Y., et al. 2019, PASP, 131, 014001

Piro, A. L., \& Morozova, V. S. 2016, ApJ, 826, 96

Piro, A. L., \& Nakar, E. 2013, ApJ, 769, 67

Piro, A. L., \& Nakar, E. 2014, ApJ, 784, 85

Piro, A. L., Chang, P., \& Weinberg, N. N. 2010, ApJ, 708, 598

Polin, A., Nugent, P., \& Kasen, D. 2019, ApJ, 873, 1

Rabinak, I., Livne, E., \& Waxman, E. 2012, ApJ, 757, 35

Salvatier, J., Wiecki, T. V., \& Fonnesbeck, C. 2016, Astrophysics Source Code

Library [record ascl:1610. 016]

Sand, D. J., Graham, M. L., Botyánszki, J., et al. 2018, ApJ, 863, 24

Schlafly, E. F., \& Finkbeiner, D. P. 2011, ApJ, 737, 103

Schlegel, D. J., Finkbeiner, D. P., \& Davis, M. 1998, ApJ, 500, 525

Shappee, B. J., Stanek, K. Z., Pogge, R. W., \& Garnavich, P. M. 2013, ApJ, 762, L5

Shappee, B. J., Piro, A. L., Holoien, T. W.-S., et al. 2016, ApJ, 826, 144

Shappee, B. J., Piro, A. L., Stanek, K. Z., et al. 2018, ApJ, 855, 6

Shappee, B. J., Holoien, T. W.-S., Drout, M. R., et al. 2019, ApJ, 870, 13

Silverman, J. M., Ganeshalingam, M., Cenko, S. B., et al. 2012, ApJ, 756, L7

Siverd, R. J., Goobar, A., Stassun, K. G., et al. 2015, ApJ, 799, 105

Smartt, S., Smith, K. W., \& Wright, D. 2014, ATel, 5850

Springob, C. M., Masters, K. L., Haynes, M. P., Giovanelli, R., \& Marinoni, C. 2007, ApJS, 172, 599

Stritzinger, M., Hamuy, M., Suntzeff, N. B., et al. 2002, AJ, 124, 2100

Stritzinger, M. D., Phillips, M. M., Boldt, L. N., et al. 2011, AJ, 142, 156

Stritzinger, M. D., Shappee, B. J., Piro, A., et al. 2018, ApJ, 864, L35

Theureau, G., Hanski, M. O., Courdreau, N., Hallet, N., \& Martin, J.-M. 2007, A\&A, 465, 71

Tomasella, L., Benetti, S., \& Tartaglia, L. 2013, ATel, 5638

Tonry, J. L., Stubbs, C. W., Lykke, K. R., et al. 2012, ApJ, 750, 99

Tucker, M. A., Shappee, B. J., \& Wisniewski, J. P. 2019, ApJ, 872, L22

Wang, X., Filippenko, A. V., Ganeshalingam, M., et al. 2009, ApJ, 699 L139

Zhang, K., Wang, X., Zhang, J.-J., et al. 2016, ApJ, 820, 67

Zheng, W., Silverman, J., Filippenko, A. V., et al. 2013, ApJ, 778, L15

Zheng, W., Shivvers, I., Filippenko, A. V., et al. 2014, ApJ, 783, L24

Zheng, W., Kelly, P. L., \& Filippenko, A. V. 2017, ApJ, 848, 66 


\section{Appendix A: Additional material}

Table A.1. Photometry of SN 2013gy and S-corrections.

\begin{tabular}{|c|c|c|c|c|}
\hline MJD & Phase $^{(a)}$ & Magnitude & S-correction & $g_{\mathrm{PS} 1}$ \\
\hline $\begin{array}{l}56631.34 \\
56631.35 \\
\end{array}$ & $\begin{array}{l}-17.16 \\
-17.15 \\
\end{array}$ & $\begin{array}{r}g_{\mathrm{PS} 1} \text { band } \\
19.483 \pm 0.079 \\
19.482 \pm 0.040 \\
\end{array}$ & $\begin{array}{l}\cdots \\
\cdots \\
\end{array}$ & $\begin{array}{l}19.483 \pm 0.079 \\
19.482 \pm 0.040 \\
\end{array}$ \\
\hline \multicolumn{5}{|c|}{$g_{\text {CSP }}$ band } \\
\hline 56633.22 & -15.28 & $18.062 \pm 0.011$ & 0.036 & $18.026 \pm 0.011$ \\
\hline 56636.26 & -12.24 & $16.622 \pm 0.009$ & 0.010 & $16.612 \pm 0.009$ \\
\hline 56637.11 & -11.39 & $16.330 \pm 0.006$ & 0.004 & $16.326 \pm 0.006$ \\
\hline 56639.11 & -9.39 & $15.769 \pm 0.008$ & -0.006 & $15.775 \pm 0.008$ \\
\hline 56640.17 & -8.33 & $15.555 \pm 0.007$ & -0.009 & $15.564 \pm 0.007$ \\
\hline 56643.15 & -5.35 & $15.104 \pm 0.008$ & -0.014 & $15.118 \pm 0.008$ \\
\hline 56644.14 & -4.36 & $15.011 \pm 0.007$ & -0.014 & $15.025 \pm 0.007$ \\
\hline 56645.13 & -3.37 & $14.938 \pm 0.007$ & -0.013 & $14.951 \pm 0.007$ \\
\hline 56646.12 & -2.38 & $14.890 \pm 0.009$ & -0.011 & $14.901 \pm 0.009$ \\
\hline 56647.16 & -1.34 & $14.857 \pm 0.007$ & -0.009 & $14.866 \pm 0.007$ \\
\hline 56648.13 & -0.37 & $14.819 \pm 0.007$ & -0.006 & $14.825 \pm 0.007$ \\
\hline 56649.11 & 0.61 & $14.826 \pm 0.006$ & -0.002 & $14.828 \pm 0.006$ \\
\hline 56650.15 & 1.65 & $14.830 \pm 0.007$ & 0.002 & $14.828 \pm 0.007$ \\
\hline 56651.10 & 2.60 & $14.850 \pm 0.008$ & 0.006 & $14.844 \pm 0.008$ \\
\hline 56652.10 & 3.60 & $14.895 \pm 0.009$ & 0.011 & $14.884 \pm 0.009$ \\
\hline 56653.13 & 4.63 & $14.925 \pm 0.005$ & 0.016 & $14.909 \pm 0.005$ \\
\hline 56654.18 & 5.68 & $14.982 \pm 0.006$ & 0.022 & $14.960 \pm 0.006$ \\
\hline 56655.19 & 6.69 & $15.040 \pm 0.009$ & 0.028 & $15.012 \pm 0.009$ \\
\hline 56656.20 & 7.70 & $15.159 \pm 0.009$ & 0.034 & $15.125 \pm 0.009$ \\
\hline 56657.21 & 8.71 & $15.202 \pm 0.010$ & 0.039 & $15.163 \pm 0.010$ \\
\hline 56658.14 & 9.64 & $15.266 \pm 0.008$ & 0.045 & $15.221 \pm 0.008$ \\
\hline 56659.15 & 10.65 & $15.357 \pm 0.006$ & 0.051 & $15.306 \pm 0.006$ \\
\hline 56660.11 & 11.61 & $15.448 \pm 0.008$ & 0.056 & $15.392 \pm 0.008$ \\
\hline 56661.16 & 12.66 & $15.534 \pm 0.007$ & 0.061 & $15.473 \pm 0.007$ \\
\hline 56662.12 & 13.62 & $15.663 \pm 0.007$ & 0.066 & $15.597 \pm 0.007$ \\
\hline 56663.11 & 14.61 & $15.741 \pm 0.007$ & 0.071 & $15.670 \pm 0.007$ \\
\hline 56664.10 & 15.60 & $15.870 \pm 0.010$ & 0.075 & $15.795 \pm 0.010$ \\
\hline 56665.08 & 16.58 & $15.955 \pm 0.008$ & 0.079 & $15.876 \pm 0.008$ \\
\hline 56666.06 & 17.56 & $16.071 \pm 0.011$ & 0.083 & $15.988 \pm 0.011$ \\
\hline 56667.07 & 18.57 & $16.154 \pm 0.010$ & 0.086 & $16.068 \pm 0.010$ \\
\hline 56669.06 & 20.56 & $16.359 \pm 0.013$ & 0.090 & $16.269 \pm 0.013$ \\
\hline 56670.14 & 21.64 & $16.491 \pm 0.013$ & 0.092 & $16.399 \pm 0.013$ \\
\hline 56671.06 & 22.56 & $16.562 \pm 0.011$ & 0.093 & $16.469 \pm 0.011$ \\
\hline 56672.12 & 23.62 & $16.662 \pm 0.009$ & 0.094 & $16.568 \pm 0.009$ \\
\hline 56673.09 & 24.59 & $16.739 \pm 0.009$ & 0.095 & $16.644 \pm 0.009$ \\
\hline 56675.05 & 26.55 & $16.893 \pm 0.008$ & 0.095 & $16.798 \pm 0.008$ \\
\hline 56676.11 & 27.61 & $16.970 \pm 0.007$ & 0.095 & $16.875 \pm 0.007$ \\
\hline 56677.04 & 28.54 & $17.033 \pm 0.009$ & 0.094 & $16.939 \pm 0.009$ \\
\hline 56678.11 & 29.61 & $17.089 \pm 0.009$ & 0.094 & $16.995 \pm 0.009$ \\
\hline 56679.05 & 30.55 & $17.166 \pm 0.006$ & 0.093 & $17.073 \pm 0.006$ \\
\hline 56680.12 & 31.62 & $17.233 \pm 0.008$ & 0.092 & $17.141 \pm 0.008$ \\
\hline 56681.05 & 32.55 & $17.293 \pm 0.008$ & 0.091 & $17.202 \pm 0.008$ \\
\hline 56684.09 & 35.59 & $17.430 \pm 0.008$ & 0.088 & $17.342 \pm 0.008$ \\
\hline 56685.07 & 36.57 & $17.484 \pm 0.008$ & 0.086 & $17.398 \pm 0.008$ \\
\hline 56686.05 & 37.55 & $17.492 \pm 0.010$ & 0.085 & $17.407 \pm 0.010$ \\
\hline 56687.06 & 38.56 & $\begin{array}{r}17.511 \pm 0.009 \\
B V .5 \text { band }\end{array}$ & 0.084 & $17.427 \pm 0.009$ \\
\hline 56690.08 & 41.58 & $17.617 \pm 0.010$ & 0.081 & $17.536 \pm 0.010$ \\
\hline 56692.08 & 43.58 & $17.674 \pm 0.011$ & 0.079 & $17.595 \pm 0.011$ \\
\hline 56694.06 & 45.56 & $17.696 \pm 0.009$ & 0.078 & $17.618 \pm 0.009$ \\
\hline 56696.07 & 47.57 & $17.733 \pm 0.012$ & 0.077 & $17.656 \pm 0.012$ \\
\hline 56703.07 & 54.57 & $17.906 \pm 0.019$ & 0.077 & $17.829 \pm 0.019$ \\
\hline 56708.06 & 59.56 & $17.968 \pm 0.011$ & 0.080 & $17.888 \pm 0.011$ \\
\hline 56632.33 & -16.17 & $18.703 \pm 0.108$ & 0.090 & $18.613 \pm 0.108$ \\
\hline 56634.31 & -14.19 & $17.477 \pm 0.053$ & 0.109 & $17.367 \pm 0.053$ \\
\hline 56635.26 & -13.24 & $17.120 \pm 0.050$ & 0.116 & $17.003 \pm 0.050$ \\
\hline 56636.28 & -12.22 & $16.725 \pm 0.025$ & 0.122 & $16.602 \pm 0.025$ \\
\hline 56637.25 & -11.25 & $16.439 \pm 0.025$ & 0.126 & $16.312 \pm 0.025$ \\
\hline 56638.29 & -10.21 & $16.125 \pm 0.022$ & 0.130 & $15.995 \pm 0.022$ \\
\hline 56639.32 & -9.18 & $15.868 \pm 0.022$ & 0.132 & $15.737 \pm 0.022$ \\
\hline 56640.29 & -8.21 & $15.647 \pm 0.029$ & 0.133 & $15.514 \pm 0.029$ \\
\hline
\end{tabular}

Notes. ${ }^{(a)}$ Temporal phase given in days with respect to the time of $B$-band maximum light on MJD $=56648.5 \pm 0.10$, i.e., 2013 December 22.

Table A.1. continued.

\begin{tabular}{crrrr}
\hline \hline MJD & Phase ${ }^{(a)}$ & Magnitude & S-correction & $g_{\text {PS } 1}$ \\
\hline 56641.38 & -7.12 & $15.426 \pm 0.025$ & 0.133 & $15.293 \pm 0.025$ \\
56645.36 & -3.14 & $15.049 \pm 0.019$ & 0.130 & $14.920 \pm 0.019$ \\
56647.32 & -1.18 & $14.994 \pm 0.014$ & 0.128 & $14.865 \pm 0.014$ \\
56648.31 & -0.19 & $14.969 \pm 0.011$ & 0.128 & $14.841 \pm 0.011$ \\
56649.30 & 0.80 & $14.966 \pm 0.011$ & 0.128 & $14.839 \pm 0.011$ \\
56650.32 & 1.82 & $14.958 \pm 0.014$ & 0.128 & $14.830 \pm 0.014$ \\
56651.27 & 2.77 & $14.996 \pm 0.011$ & 0.128 & $14.868 \pm 0.011$ \\
56652.26 & 3.76 & $15.028 \pm 0.014$ & 0.127 & $14.901 \pm 0.014$ \\
56653.28 & 4.78 & $15.051 \pm 0.014$ & 0.127 & $14.924 \pm 0.014$ \\
56654.26 & 5.76 & $15.098 \pm 0.011$ & 0.126 & $14.972 \pm 0.011$ \\
56655.21 & 6.71 & $15.160 \pm 0.011$ & 0.124 & $15.036 \pm 0.011$ \\
56656.28 & 7.78 & $15.214 \pm 0.015$ & 0.122 & $15.093 \pm 0.015$ \\
56657.23 & 8.73 & $15.280 \pm 0.015$ & 0.118 & $15.162 \pm 0.015$ \\
56658.24 & 9.74 & $15.358 \pm 0.015$ & 0.114 & $15.244 \pm 0.015$ \\
56659.25 & 10.75 & $15.435 \pm 0.015$ & 0.109 & $15.326 \pm 0.015$ \\
56660.21 & 11.71 & $15.523 \pm 0.025$ & 0.104 & $15.419 \pm 0.025$ \\
56661.23 & 12.73 & $15.601 \pm 0.032$ & 0.097 & $15.504 \pm 0.032$ \\
56663.22 & 14.72 & $15.782 \pm 0.017$ & 0.081 & $15.701 \pm 0.017$ \\
56665.23 & 16.73 & $15.959 \pm 0.023$ & 0.063 & $15.896 \pm 0.023$ \\
56667.24 & 18.74 & $16.110 \pm 0.031$ & 0.032 & $16.078 \pm 0.031$ \\
56672.25 & 23.75 & $16.470 \pm 0.045$ & -0.110 & $16.580 \pm 0.045$ \\
56674.24 & 25.74 & $16.596 \pm 0.029$ & -0.136 & $16.732 \pm 0.029$ \\
56676.23 & 27.73 & $16.727 \pm 0.030$ & -0.151 & $16.878 \pm 0.030$ \\
56678.22 & 29.72 & $16.845 \pm 0.028$ & -0.161 & $17.006 \pm 0.028$ \\
56680.20 & 31.70 & $16.986 \pm 0.033$ & -0.166 & $17.152 \pm 0.033$ \\
56682.19 & 33.69 & $17.086 \pm 0.030$ & -0.168 & $17.254 \pm 0.030$ \\
56684.19 & 35.69 & $17.192 \pm 0.032$ & -0.167 & $17.358 \pm 0.032$ \\
56689.16 & 40.66 & $17.389 \pm 0.054$ & -0.153 & $17.542 \pm 0.054$ \\
56702.14 & 53.64 & $17.824 \pm 0.111$ & -0.085 & $17.909 \pm 0.111$ \\
\hline & & & &
\end{tabular}


Table A.2. Journal of spectroscopic observations.

\begin{tabular}{|c|c|c|c|c|c|}
\hline Date & MJD & Phase $^{(a)}$ & Telescope ${ }^{(b)}$ & Instrument & $-v_{\mathrm{abs}} \operatorname{Si~II} \lambda 6355\left(\mathrm{~km} \mathrm{~s}^{-1}\right)$ \\
\hline 2013 Dec 07.5 & 56633.5 & -15.0 & FTS & FLOYDS $^{c}$ & $14039_{-104}^{+198}$ \\
\hline 2013 Dec 07.7 & 56633.7 & -14.8 & LJT & YFOSC & $13709_{-151}^{+274}$ \\
\hline 2013 Dec 07.8 & 56633.8 & -14.7 & Copernico & AFOSC & $13732_{-132}^{+510}$ \\
\hline 2013 Dec 08.5 & 56634.5 & -14.0 & FTS & FLOYDS $^{c}$ & $13756_{-71}^{+90}$ \\
\hline 2013 Dec 08.8 & 56634.8 & -13.7 & LJT & YFOSC & $13322_{-142}^{+326}$ \\
\hline 2013 Dec 09.7 & 56635.7 & -12.8 & LJT & YFOSC & $13067_{-61}^{+170}$ \\
\hline 2013 Dec 14.0 & 56640.0 & -8.5 & Magellan Clay & MagE & $11509_{-33}^{+76}$ \\
\hline 2013 Dec 15.4 & 56641.4 & -7.1 & FTS & FLOYDS $^{c}$ & $11174_{-66}^{+146}$ \\
\hline 2013 Dec 17.4 & 56643.4 & -5.1 & FTS & FLOYDS $^{c}$ & $10645_{-28}^{+28}$ \\
\hline 2013 Dec 20.5 & 56646.5 & -2.0 & FTS & FLOYDS $^{c}$ & $10673_{-24}^{+222}$ \\
\hline 2013 Dec 20.7 & 56646.7 & -1.8 & LJT & YFOSC & $10631_{-66}^{+52}$ \\
\hline 2013 Dec 21.7 & 56647.7 & -0.8 & LJT & YFOSC & $10178_{-47}^{+90}$ \\
\hline 2013 Dec 25.8 & 56651.8 & +3.3 & NOT & ALFOSC & $10149_{-71}^{+90}$ \\
\hline $2013 \operatorname{Dec} 27.7$ & 56653.7 & +5.2 & XLT & BFOSC & $9862_{-85}^{+411}$ \\
\hline 2013 Dec 28.3 & 56654.3 & +5.8 & Keck & LRIS & $9913_{-14}^{+71}$ \\
\hline $2013 \operatorname{Dec} 31.2$ & 56657.2 & +8.7 & Magellan Baade & IMACS & $9734_{-57}^{+94}$ \\
\hline 2013 Dec 31.7 & 56657.7 & +9.2 & LJT & YFOSC & $9682_{-57}^{+47}$ \\
\hline 2014 Jan 03.6 & 56660.6 & +12.1 & XLT & BFOSC & $10971_{-850}^{+836}$ \\
\hline 2014 Jan 07.6 & 56664.6 & +16.1 & LJT & YFOSC & $9267_{-94}^{+170}$ \\
\hline 2014 Jan 08.3 & 56665.3 & +16.8 & $d u$ Pont & WFCCD & $9059_{-99}^{+99}$ \\
\hline 2014 Jan 14.0 & 56671.0 & +22.5 & NOT & ALFOSC & $8705_{-123}^{+302}$ \\
\hline 2014 Jan 23.3 & 56680.3 & +31.8 & Shane & Kast & $8134_{-33}^{-194}$ \\
\hline 2014 Feb 09.9 & 56697.9 & +49.4 & NOT & ALFOSC & $7487_{-482}^{+439}$ \\
\hline 2014 Aug 14.5 & 56883.5 & +235.0 & Magellan Baade & IMACS & $\ldots$ \\
\hline
\end{tabular}

Notes. ${ }^{(a)}$ Temporal phase given in days with respect to the time of $B$-band maximum light on MJD $=56648.5 \pm 0.10$, i.e., 2013 December 22.break ${ }^{(b)}$ FTS $=2.0 \mathrm{~m}$ Faulkes Telescope South, LJT $=2.4 \mathrm{~m}$ Li-Jiang Telescope, Copernico $=$ INAF Osservatorio Astronomico di Padova $1.8 \mathrm{~m}$ Telescope, NOT $=2.5 \mathrm{~m}$ Nordic Optical Telescope, XLT $=2.6 \mathrm{~m}$ Xing-Long Telescope. ${ }^{(c)}$ Spectra were published by Graham et al. $(2017)$.

Table A.3. Key parameters of SN 2013gy.

\begin{tabular}{ccccccccc}
\hline \hline $\begin{array}{c}t_{B \max } \\
{[\mathrm{MJD}]}\end{array}$ & $\begin{array}{c}\Delta m_{15}(B) \\
{[\mathrm{mag}]}\end{array}$ & $s_{B V}$ & $\begin{array}{c}E(B-V)_{\text {tot }} \\
{[\mathrm{mag}]}\end{array}$ & $\begin{array}{c}\mu^{(a)} \\
{[\mathrm{mag}]}\end{array}$ & $\begin{array}{c}M_{B} \\
{[\mathrm{mag}]}\end{array}$ & $\begin{array}{c}t_{\text {first }} \\
{[\mathrm{MJD}]}\end{array}$ & $\begin{array}{c}\alpha \\
t_{\text {rise }} \\
{[\mathrm{days}]}\end{array}$ \\
\hline $56648.5 \pm 0.10$ & $1.234 \pm 0.060$ & $0.892 \pm 0.05$ & $0.106 \pm 0.060$ & $33.75 \pm 0.15$ & $-19.32 \pm 0.16$ & $56629.4 \pm 0.13$ & $2.16_{-0.06}^{+0.06}$ & $19.10_{-0.13}^{+0.12}$ \\
\hline
\end{tabular}

Notes. ${ }^{(a)}$ Based on red-shift assuming $H_{0}=73 \mathrm{~km} \mathrm{~s}^{-1}$ and adopting a correction based on a Virgo, Great Attractor and Shapley infall model.

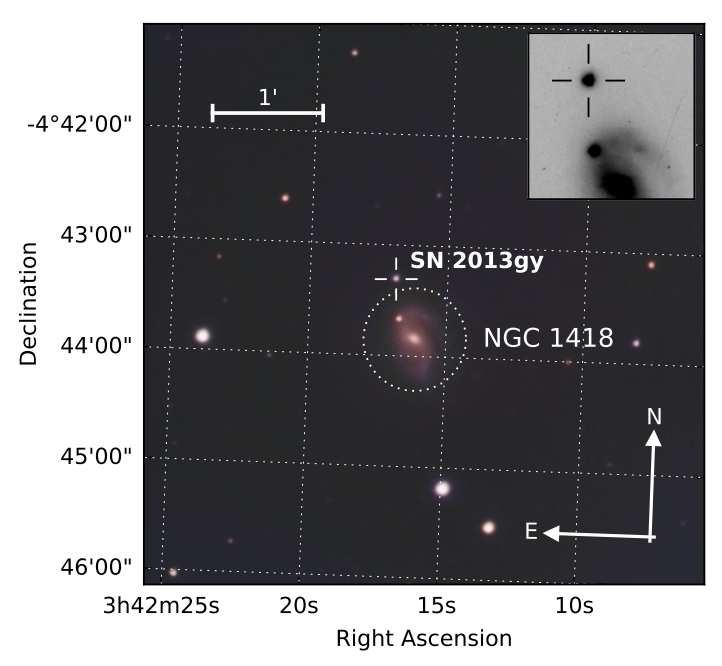

Fig. A.1. Composite image of NGC 1418 (north up and east left) constructed from multiband images obtained with the Swope telescope. The inset contains a close-up view of the position of SN 2013gy, highlighting its position in the outer region of the host. 


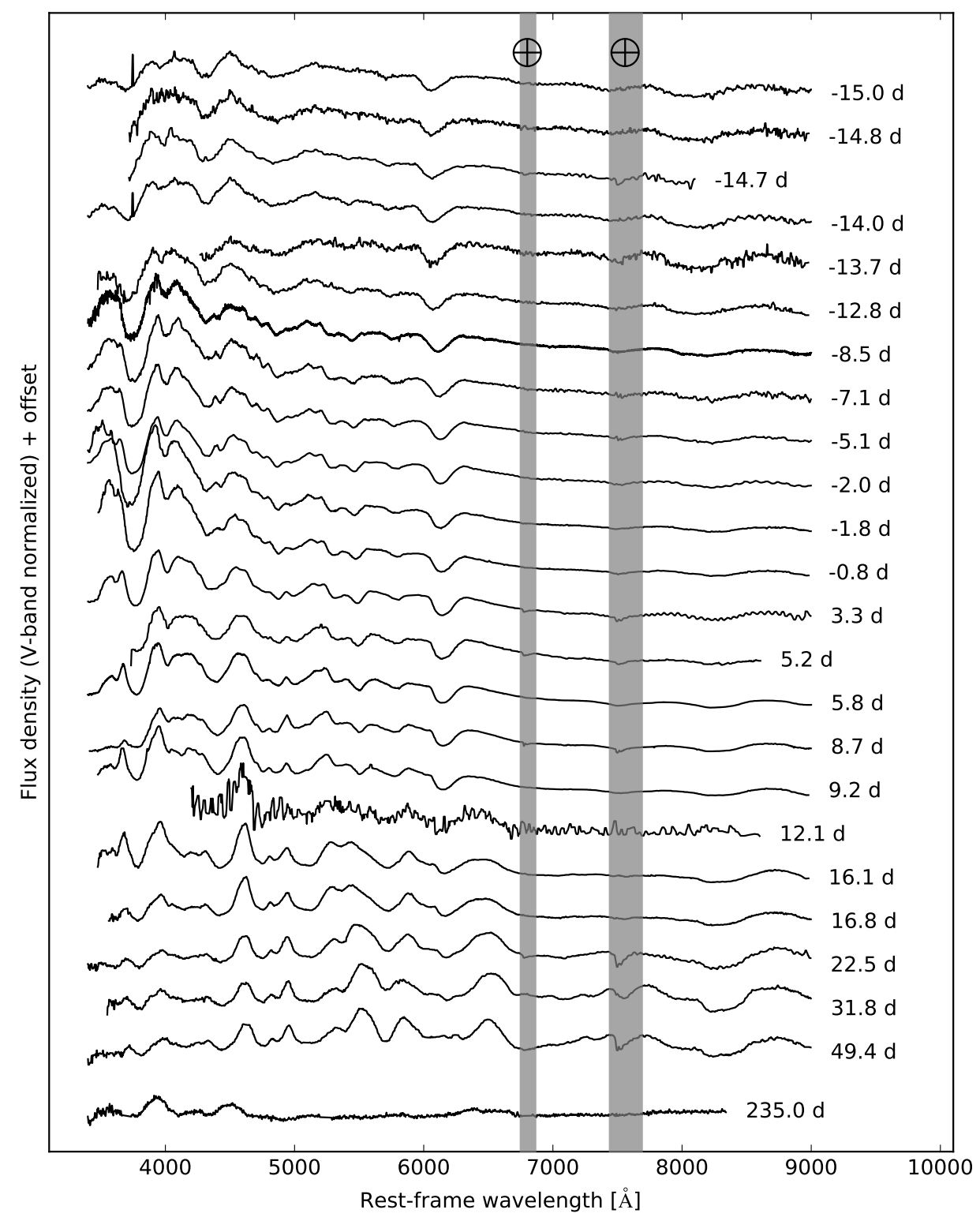

Fig. A.2. Spectroscopic time series of SN 2013gy used to compute S-corrections, enabling us to transform natural-system $g_{\mathrm{CSP}}-$ and $B V .5$-band photometry to the $g_{\mathrm{PS} 1}$ natural system. 